\section{PS-114 PROGNOSTIC PERFORMANCE OF PROTON MAGNETIC RESONANCE SPECTROSCOPY (HMRS) METABOLITE RATIOS IN THE FIRST 96 HOURS OF LIFE IN ASPHYXIATED NEONATES}

${ }^{1} \mathrm{H}$ Barta, ${ }^{1} \mathrm{~A}$ Jermendy, ${ }^{1} \mathrm{M}$ Kolossvary, ${ }^{2} \mathrm{G}$ Rudas, ${ }^{1} \mathrm{M}$ Szabo. ${ }^{1} 1 \mathrm{st}$ Department of Pediatrics, Semmelweis University, Budapest, Hungary; ${ }^{2} M R$ Research Center, Semmelweis University, Budapest, Hungary

10.1136/archdischild-2014-307384.408

Background and aims Neonatal hypoxic-ischaemic encephalopathy (HIE) can lead to neurodevelopmental impairment, raising a need for early prognostic tools to guide therapy. The prognostic value of HMRS performed between 5-30 days of life has been extensively studied, although few studies focus on earlier periods. Therefore, we investigated the prognostic performance of HMRS during the first $96 \mathrm{~h}$ of life.

Methods 55 consecutive hypothermia-treated HIE neonates were examined by HMRS at three echo-times (TE = $35 \mathrm{~ms}, 144 \mathrm{~ms}$, $288 \mathrm{~ms}$ ) between $6-96 \mathrm{~h}$ of age, depending of clinical stability. Patients were divided into favourable $(\mathrm{n}=38)$ and unfavourable ( $n=17$ ) outcome groups based on Bayley II MDI and PDI scores ( $\geq 70$ vs $<70$ or death, respectively) assessed at $18-26$ months of age. Associations between 36 routinely measured metabolite ratios (MROs) and outcome were studied. The prognostic performance of MROs was evaluated by ROC analysis. Time-dependent changes of MROs in whole patient population were also assessed. Results 6 MROs showed significant difference between outcome groups after correction for multiple testing $(\mathrm{p}<0.0014)$. ROC analysis revealed that Myo-inositol/N-Acetyl-Aspartate $(\mathrm{TE}=$ 35 ) gives best prediction for outcome with $85.71 \%$ sensitivity and $91.30 \%$ specificity. Assessment of time-dependency showed that 4 of 6 MROs stay constant during the first $96 \mathrm{~h}$ of life, all containing Myo-inositol.

Conclusions Our findings suggest that during the first $96 \mathrm{~h}$ of life HMRS could be a useful early prognostic tool in predicting the outcome of asphyxiated neonates. Myo-inositol/N-Acetyl-Aspartate ratio was found to be the best and time-independent predictor.

\section{PS-115 1H-NMR MEASURMENT OF CORD GLYCEROL SUCCINATE PREDICTS SEVERE ENCEPHALOPATHY AND DEATH IN NEONATAL HYPOXIC-ISCHAEMIC ENCEPHALOPATHY}

${ }^{1} \mathrm{C}$ Ahearne, ${ }^{1} \mathrm{NM}$ Denihan, ${ }^{1} \mathrm{BH}$ Walsh, ${ }^{2} \mathrm{SN}$ Reinke, ${ }^{3} \mathrm{BD}$ Sykes, ${ }^{4} \mathrm{LC}$ Kenny, ${ }^{5} \mathrm{DI}$ Broadhurst, ${ }_{1}^{1} \mathrm{~GB}$ Boylan, ${ }^{1} \mathrm{DM}$ Murray. ${ }^{1}$ Neonatal Brain Research Group (NBRG) and Irish Centre for Fetal and Neonatal Translational Research (INFANT), University College Cork, Cork, Ireland; ${ }^{2}$ Department of Medicine and Biochemistry, University of Alberta, Edmonton, Canada; ${ }^{3}$ Department of Biochemistry, University of Alberta, Edmonton, Canada; ${ }^{4}$ Irish Centre for Fetal and Neonatal Translational Research, University College Cork, Cork, Ireland; ${ }^{5}$ Department of Medicine, University of Alberta, Edmonton, Canada

\subsection{6/archdischild-2014-307384.409}

Background and aims The outcome of infants with severe hypoxic-ischaemic encephalopathy (HIE) remains extremely poor. Early identification of these infants could improve patient management and direct care. Our aim was to correlate the metabolomic profile of umbilical cord blood (UCB) with outcome in neonatal HIE.

Methods Full term infants with perinatal asphyxia and healthy matched controls were recruited from 2009 to 2011. All had UCB biobanked at $-80^{\circ} \mathrm{C}$ within $3 \mathrm{~h}$ of birth and multichannel electroencephalogram (EEG) recorded in the first $24 \mathrm{~h}$ of life. The metabolite profile of UCB was analysed using nuclear magnetic resonance (NMR) spectroscopy. Infant outcome was assessed using the Bayley Scales of Infant and Toddler development at 3 years.

Results The UCB metabolomic profile of 118 infants was described; 59 healthy controls, 34 perinatal asphyxia (no HIE) and 25 HIE defined by Sarnat score and EEG (13 mild, 6 moderate, 6 severe). Of the 6 cases of severe HIE, at 3 years; 4 have died, 1 survived with severe dyskinetic cerebral palsy and 1 had a normal outcome. A characteristic pattern of raised glycerol + succinate occurred in those infants with severe encephalopathy and very low voltage EEG $\left(\mathrm{R}^{2}=0.49, \mathrm{p}<0.001\right)$.

Conclusion Alterations in glycerol and succinate at birth reflect critical energy failure in infants with severe neuronal injury. This measurement at birth could help clinicians to identify infants who will not benefit from standard neuroprotection and may need experimental intervention, or limitation of care.

\section{PS-116 WITHDRAWN}

\section{PS-117 PRETERM CEREBRAL MICROCIRCULATION ASSESSED WITH COLOUR DOPPLER: A PILOT STUDY}

M Raets, P Govaert, TG Goos, IKM Reiss, RCJ de Jonge, J Dudink. Neonatology, Erasmus University Medical Center - Sophia Children's Hospital, Rotterdam, Netherlands

\subsection{6/archdischild-2014-307384.410}

Background and aims Pilot study to explore feasibility of a colour Doppler technique for monitoring cerebral perfusion at the level of microvessels.

Methods Between March 1st, 2011, and January 30th, 2013, all admitted infants born before 29 weeks of gestation were eligible for Doppler imaging. Perfusion images were acquired in a standard coronal plane. Image quality was assessed by two authors (MR, PG). The region of interest (ROI) was manually selected. A segmentation tool was developed to separate colour data from the greyscale 2D images, leading to a percentage and number of colour pixels in the image (Doppler colour index; DCI). Intraand interobserver agreement was analysed.

Results Intra- and interobserver agreement for placement of ROIs was good (bias -0.24 resp. -0.74 percentage points). Colour Doppler was able to depict microvessels in cortex, white matter and deep grey matter. The median DCI in a region of cortex-white matter was $7.8 \%$ with a wide range (1.4\%-25.6\%). There was no significant difference between the left and right hemisphere (Mann-Whitney U, P-value 0.61). Clinically relevant observations were tabulated, e.g. distant effect of GMH on regional perfusion.

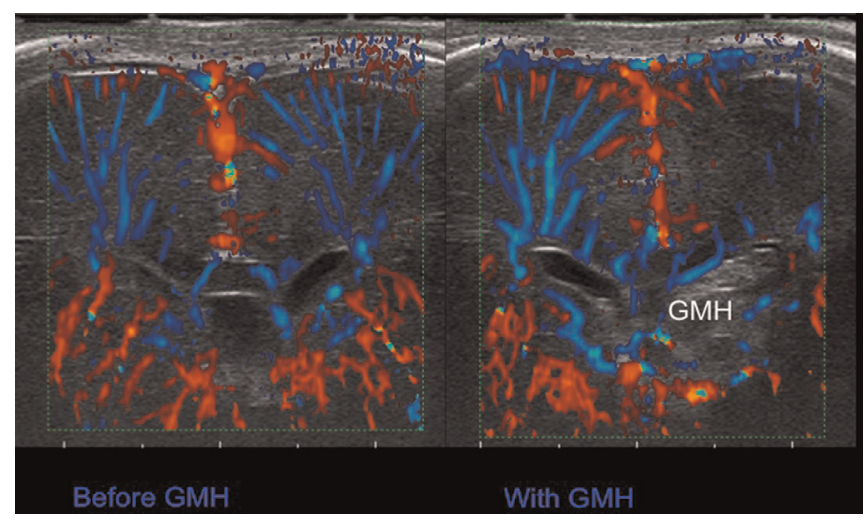

Abstract PS-117 Figure 1 
Conclusion Sonographic small vessel visualisation may help understand pathogenetic mechanisms related to perfusion and is valuable to monitor effects of treatment.

\section{PS-117a HYPEROXIA REDUCES ASTROGLIAL PDGF EXPRESSION AND IMPAIRS OLIGODENDROGLIAL DEVELOPMENT IN THE CEREBELLUM}

${ }^{1} \mathrm{~T}$ Scheuer, 'V Brockmöller, ${ }^{2} \mathrm{MB}$ Knowiton, ${ }^{3} \mathrm{~S}$ Müller, ${ }^{1} \mathrm{C}$ Bührer, ${ }^{1} \mathrm{~S}$ Endesfelder, ${ }^{1} \mathrm{~T}$ Schmitz. 'Neonatology, Charité University Medicine, Berlin, Germany; ${ }^{2}$ Newborn Medicine, Children's Hospital of Pittsburgh, Pittsburgh PA, USA; ${ }^{3}$ Center for Stroke Research, Charité University Medicine, Berlin, Germany

10.1136/archdischild-2014-307384.411

Although brain injury of preterm infants has widely been ascribed to the cerebrum, recent studies demonstrate that injury of the cerebellum often occurs, too. However, mechanisms of the cerebellar injury are hardly understood. In general, hypoxiaischemia, infection/inflammation, and hyperoxia are supposed to be toxic stimuli in the immature brain.

We used a hyperoxia model established in rats by using $80 \%$ $\mathrm{O}_{2}$ exposure for $24 \mathrm{~h}$ from $\mathrm{P} 6$ to $\mathrm{P} 7$, and determined development and maturation of oligodendroglial cells in the cerebellum during recovery in room air until P30. Expression of platelet derived growth factor (PDGF)-A as a crucial factor for oligodendroglial development was determined in cerebellar tissue and in cultures of cerebellar astroglia.

Volume of the cerebellum measured by MRI was significantly reduced in P30 rats after hyperoxia as compared to control litters $(208$ vs. $232 \mu \mathrm{L}$ respectively, $\mathrm{p}<0.05)$. Myelination measured by MBP in immunostainings and Western blots was significantly reduced during recovery from P9 to P30. Numbers of NG2+TUNEL+ oligodendroglial precursor cells were increased by hyperoxia, and oligodendroglial maturation towards $\mathrm{CC} 1+$ stages was significantly diminished. Expression of PDGFA was reduced after hyperoxia by $46 \%$ at P7 and by $27 \%$ at P30 $(\mathrm{n}=4 ; \mathrm{p}<0.01$ each). In astrocyte cultures, exposure to $80 \%$ $\mathrm{O}_{2}$ also caused a significant downregulation of PDGF-A expression by $42 \%$ ( $\mathrm{n}=6$; $\mathrm{p}<0.01)$.

Hyperoxia resembles critical features of the cerebellar pathology found in preterm infants. Astroglial changes caused by hyperoxia include reduced PDGF synthesis and are likely to contribute to oligodendroglial damage in the cerebellum.

\section{PS-117b STUDY OF DEFAULT MODE NETWORK IN FUNCTIONAL MAGNETIC RESONANCE IMAGING BETWEEN PREMATURE AND FULL-TERM INFANTS}

SFeng. Pediatrics, Daping Hospital Third Military Medical University, Chongqing, China

\subsection{6/archdischild-2014-307384.412}

Objective To explore the differences in default mode network between premature and full-term infants by using resting-state functional magnetic resonance imaging (RS-fMRI).

Methods 17 premature infants and 27 full-term infants were subject to RS-fMRI scanning. The data were analysed by amplitude of low frequency fluctuation (ALFF), while relative to the full term infants, the enhanced and weakened regions of ALFF in premature infants were observed.

Results The resting-state fMRI indicated that premature infants showed significant declines of ALFF in the medial prefrontal cortex (MPFC), inferior frontal gyrus (IFG), inferior orbitofrontal
Abstract PS-117b Table 1 Group information of the premature and full term infants

\begin{tabular}{llll}
\hline & $\begin{array}{l}\text { Premature }(\mathrm{N}=17) \\
\text { (Mean/SD) }\end{array}$ & $\begin{array}{l}\text { Full-term }(\mathrm{N}=29) \\
(\text { Mean/SD) }\end{array}$ & $\mathrm{p}$ Value \\
\hline Gender & $11 \mathrm{M} / 6 \mathrm{~F}$ & $17 \mathrm{M} / 12 \mathrm{~F}$ & $\mathrm{~N} / \mathrm{A}$ \\
GA [weeks \pm days] & $33.8 \pm(1.77$ days $)$ & $38.7 \pm(1.09$ days $)$ & $\mathrm{p}<0.05$ \\
Age [days] & $2.85 \pm 7.07$ & $5.27 \pm 8.35$ & $\mathrm{p}>0.05$ \\
\hline
\end{tabular}

Values are mean \pm standard error.

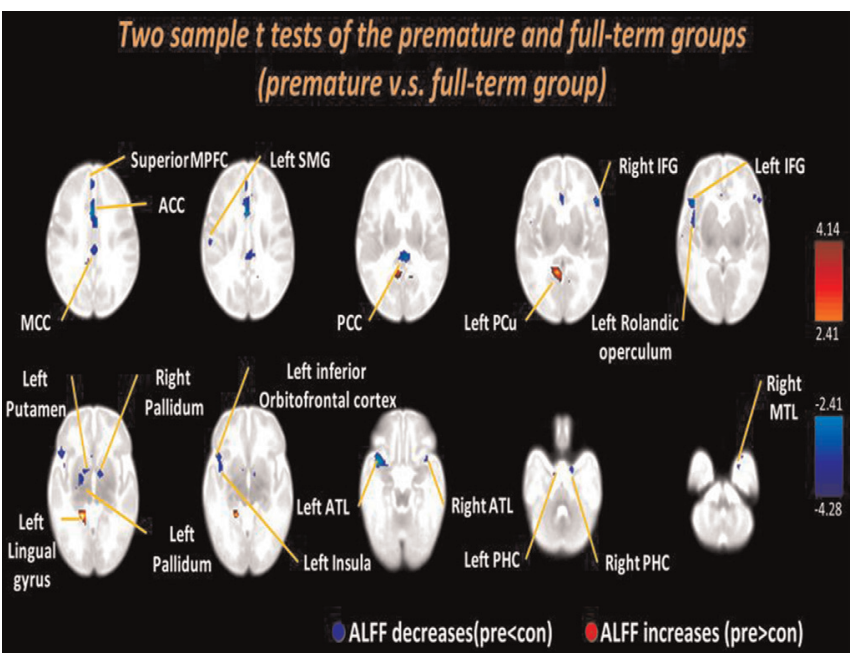

Abstract PS-117b Figure 1 Two sample t-tests of the premature and full-term group (premature v.s. full-term, no correction, $p<0.01$ ). Brain areas exhibited significant decreased ALFF included the MPFC, IFG,

cortex, supramarginal (SMG), insula, anterior temporal lobe (ATL), middle temporal lobe (MTL), anterior cingulate cortex (ACC), posterior cingulate cortex (PCC), middle cingulate cortex (MCC), pallidum, putamen, rolandic operculum, parahippocampalcortex (PHC). And significant increases in precuneus $(\mathrm{PCu})$, fusiform and calcarine cortex were noted.

Conclusions Compared with full-term infants, we speculated that premature infants might present a development delay in the frontal, temporal and middle cingulate gyrus. The decreased ALFF in the inferior frontal might be connected with cognitive deficits or delay cognitive development of some premature children. Another interesting area lied in the middle cingulate gyrus which was related with human's self-consciousness, which implied that there might be some problems of self-consciousness of premature infants. The increased effects on the premature infants might be because of the compensation effect.

Abbreviation: GA: gestational age; M: male; F: female.

\section{Improvement of Clinical Practice}

\section{PS-118 DISCERNING THE IMPACT OF THE INTERNSHIP EXPERIENCE FOR THE BASIC SPECIALIST TRAINEE IN PAEDIATRICS (BSTP)}

G King, E Clarke, O'NM Talbot. Department of Paediatrics, Mayo General Hospital, Republic of Ireland

10.1136/archdischild-2014-307384.413 